\title{
Design of a hybrid honeycomb unit cell with enhanced in -plane mechanical properties
}

\author{
Sajjad Raeisi ${ }^{1}$, Prasad Tapkir ${ }^{2}$, Farha Ansari ${ }^{2}$, and Andres Tovar ${ }^{2}$ \\ 1- Purdue University \\ 2- Indiana University - Purdue University Indianapolis
}

\begin{abstract}
Sandwich structures with honeycomb core are widely used in the lightweight design and impact energy absorption applications in automotive, sporting, and aerospace industries. Recently, the auxetic honeycombs with negative Poisson's ratio attract a substantial attention for different engineering products. In this study, we implement Additive Manufacturing technology, experimental testing, and Finite Element Analysis (FEA) to design and investigate the mechanical behavior of a novel unit cell for sandwich structure core. The new core model contains the conventional and auxetic honeycomb cells beside each other to create a Hybrid Honeycomb (HHC) for the sandwich structure. The different designs of unit cells with the same volume fraction of $15 \%$ are 3D-printed using Fused Deposition Modeling technique, and the comparative study on the mechanical behavior of conventional honeycomb, auxetic honeycomb, and HHC structures is conducted. The quasi-static uniaxial compression tests are performed on the printed samples to investigate the mechanical behavior of the printed structures. The deformation and failure modes of the different designs are studied at the cell level utilizing FEA of the compression test and experimental observation. The compressive strength of the different design is measured using three experimental tests. The new HHC unit cell design shows significantly higher mechanical properties than the auxetic and the conventional designs. Modifying the design variables of hybrid cellular core structure allows us to tailor the mechanical properties and deformation pattern in macro level to achieve the desired mechanical properties in sandwich structures.
\end{abstract}

\section{Introduction}

The natural cellular architectures are the one of the evolutionary solutions to design the structures with high ratio of stiffness to density. Lightweight structures are nowadays increasingly used for different engineering applications such as aerospace, automobile, biomedical, marine, windmill etc. The bio-inspired honeycomb sandwich structures are a group of lightweight material, which deliver excellent multifunctional properties such as high flexural stiffness, thermal insulation and high energy-absorption capabilities [1-9].

The previous studies illustrate that the stiffness and strength of the honeycomb structures in out-of-plane direction is significantly high due to the enormous deformations of the cell walls in axial loading direction $[1,10,11]$. On the other hand, due to the bending of the cells, the mechanical properties of the honeycomb structures in an in-plane directions are much lower [1]. To address this trade-off, several research studies are conducted to enhance the in-plane mechanical properties of the honeycomb structures by changing the cell angles of the honeycombs, to tailor the in-plane properties [1, 12]. Another alternative approach to address these properties is the implementation of sandwich structures into mechanical applications. A sandwich structure is a core having opposite top and bottom surfaces, a plurality of discrete cellular structure is disposed through the core, which extends up to the top and bottom surfaces of the structure to secure the cellular core and to improve the strength of the structure [13]. To gain the preliminary insight about the mechanical properties of sandwich structures, the finite element models as well as physical experiments of sandwich structures are previously studied through mechanical tests such as multiple failure modes tests and three-point bending tests [14, 15]. Furthermore, the role of the core of sandwich structures was studied on the lattice core sandwich panel, where end frames and the struts along with unidirectional fibers strengthened the core [16]. The out of plane compression tests were conducted in the corresponding research to demonstrate the strength of the composite pyramidal lattice truss structure with the improved core properties. This in turn highlights the vital role of the core to tailor the mechanical properties of sandwich structures.

Besides the high strength requirements of sandwich structures, it is also desired to maintain, as low structural weight as possible. In such cases, sandwich structures with honeycomb core designs are more suitable as the honeycomb designs possess high flexural stiffness to weight ratio compared to the monocoque and other structures. Additionally, these structures also have excellent mechanical energy absorption ability and a high crush strength. In the application of sandwich structures. to further improve these mechanical properties of honeycomb designs, Zhou, H., et al., tested the sandwich structures of two Nomex honeycombs with multiple combinations with or without the clapboards [17]. Their study concluded that the energy absorption of the combination structure with clapboard was greater than that of the combination without clapboards. Another innovative approach of improving the designs of sandwich core along with the conventional honeycomb structure was proposed by S. Raeisi and A. Tovar [18]. The corresponding research developed sandwich designs with method of topology optimization of unit cells, which in turn generated heterogeneous cellular structures inspired by close-cell aluminum foam with minimum structural compliance to the mechanical loading.

In recent years, honeycomb structures with negative Poisson's ratio (NPR), technically termed as auxetic structures, have become an attractive research topic. This type of structures offer the property of being thicker in dimension perpendicular to the direction of stretching and vice-versa, which in turn also provides mechanical properties such

This is the author's manuscript of the article published in final edited form as: 
as enhanced shear modulus, indentation resistance, and improved fracture toughness. Interestingly, the auxetic structures find their application in multiple engineering domain due to the application specific properties such as enhanced resistance to buckling under pure bending, enhanced acoustic absorption capacity, and tendency to have the formation of double curvature under flexure. Ingrole, A., et al. [11] demonstrated some of these extraordinary properties of auxetic structures through the comparative study of in-plane uniaxial compression loading behavior of hybrid structure combining regular auxetic strut and other conversional designs.

Altogether, with such a wide range of structural properties, auxetic structures have explored applications from atomic scale to macro scale and in a vast domain ranging from engineering to bio-medical technology. Some of the specific application are namely, adaptive and deployable structures, MEMS devices, filters and sieves, seat cushion material, energy absorption components, viscoelastic damping materials, fastening devices, strain amplifiers, piezoelectric devices, and structural health monitoring components. However, with high mass density, auxetic structures are prone to pose problems in some of the applications due to the closed-cell architecture, including moisture, gas retention, thermal conductivity, and limited improvements in strength versus relative density.

In this study, we propose a new hybrid cellular design, by combining the conventional and auxetic cell, for employing as sandwich structures core. The mechanical properties of the proposed hybrid cell are compared to those of the conventional and auxetic unit cell structures. The in-plane mechanical performance of the hybrid structures is assessed with their deformation mechanisms in cell level under quasi-static uniaxial compressive loading. Also, Finite Element Analysis (FEA) is utilized to compare the mechanical properties of the sandwich structures with different cell architectures. The numerical simulation results are validated by using experimental tests carried out on the samples manufactured by $3 \mathrm{D}$ printing. The numerical simulations are in a good agreement with experimental results.

\section{Design methodology}

\section{Unit cell design}

In this research three different unit cells are designed and studied. The three different unit cells are known as Conventional honeycomb (CHC), Auxetic Honeycomb (AHC) and the Hybrid honeycomb (HHC). The $\mathrm{CHC}$ and $\mathrm{AHC}$ are the very popular structures with positive and negative Poisson's ratio values, respectively. The introduced hybrid unit cell is designed by merging the previous cells (CHC and $\mathrm{AHC}$ ) to obtain a structure with approximately zero Poison's ratio. The unit cells are designed with the same relative density of $15 \%$. The volume fraction of each unit cell is calculated based on uniform thickness distribution on its struts. The design parameters of each unit cell and corresponding calculation material properties are further discussed in the next sections.

\section{Conventional Honeycomb}

The conventional honeycomb is the unit cell with positive Poison's ratio. The design parameters are required to design and calculate the ratio between mechanical properties of the conventional honeycomb

Page 2 of 8 and the base solid material. The representation of the $\mathrm{CHC}$ unit cell and corresponding design parameters are shown in Figure 1. The design parameters are as follows; $h$ is the length of the horizontal strut, 1 is the length of the inclined struts and, $\theta$ is the angle of the inclined strut, and $t$ is the thickness of the strut. The angle $\theta$ is positive for the honeycomb structure and negative for auxetic honeycomb structure.

The relative density of the honeycomb is defined as ratio between the area of the struts and the entire area of the cell (the solid cell). The relative density is the function of design.

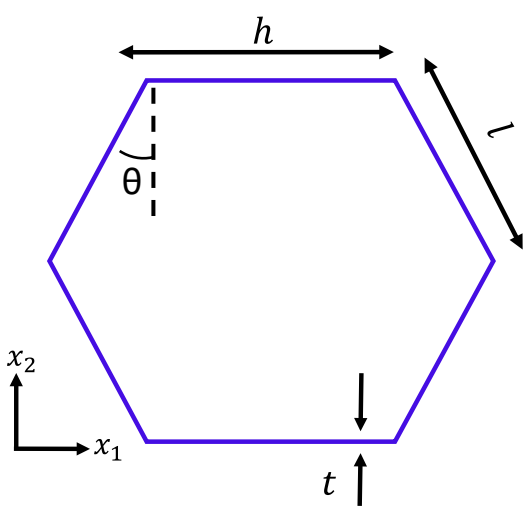

Figure 1: Design parameters of Conventional Honeycomb.

The relative density of the conventional honeycomb and auxetic honeycomb for low relative density values (that $t / l$ is small) is defined based on (1).

$$
\frac{\rho^{*}}{\rho_{s}}=\frac{\mathrm{t}\left(\frac{h}{l}+2\right)}{2 \cos \theta\left(\frac{h}{l}+\sin \theta\right)}
$$

Where $\rho^{*}$ is the density of the honeycomb and $\rho_{S}$ is the density of the solid material which struts are made from. The Poisson's ratio of the honeycomb structures under uniaxial loading is derived by Gibson and Ashby [1]. The analytical expression of Poisson's ratio of the honeycomb for in-plane directions $\left(v_{21}{ }^{*}\right.$ and $\left.v_{12}{ }^{*}\right)$ are given in (2) and (3).

$$
v_{12}{ }^{*}=-\frac{\epsilon_{1}}{\epsilon_{2}}=\frac{\left(\frac{h}{l}+\sin \theta\right) \sin \theta}{(\cos \theta)^{2}}
$$

And for the case of loading in $\boldsymbol{x}_{\mathbf{2}}$ direction the Poisson's ratio is expressed as:

$$
v_{21}{ }^{*}=-\frac{\epsilon_{2}}{\epsilon_{1}}=\frac{(\cos \theta)^{2}}{\left(\frac{h}{l}+\sin \theta\right) \sin \theta}
$$

\section{Auxetic Honeycomb}

The auxetic honeycomb is the unit cell with negative Poison's ratio. All the given design parameters and equations for relative density and in-plane Poisson's ratio values of the conventional honeycomb are 
valid for the auxetic unit cell. However, the only difference between the conventional honeycomb and auxetic honeycomb is the angle between the inclined struts and horizontal line which is perpendicular to the vertical struts. The graphical representation of auxetic unit cell and design parameters are shown in Figure 2.

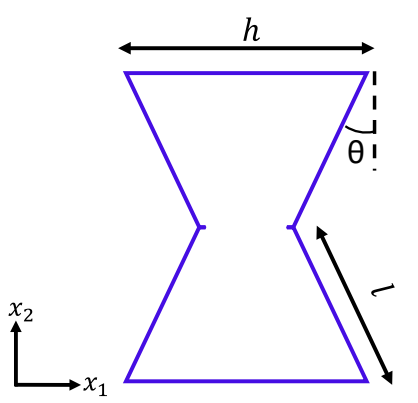

Figure 2: Design parameters of Auxetic Honeycomb.

\section{Hybrid honeycomb}

The hybrid honeycomb is the introduced geometry for unit cell which is a combination of conventional and auxetic structures with similar material. The hybrid honeycomb unit cell is designed by assembling an auxetic unit cell inside a conventional unit cell. The design parameter of the hybrid unit cell is $l, h, t_{1}, t_{2}, \theta$, and $\gamma$. The two base unit cells ( $\mathrm{CHC}$ and $\mathrm{AHC}$ ) have the same design parameters $l, w$ and $h$. Figure 3 represents the assembled $\mathrm{CHC}$ and $\mathrm{AHC}$ unit cells in red and blue colors, respectively. In addition, black horizontal inclined struts are added to connect the elbows of CHC to those of the AHC. These black struts are designed to translate the vertical compression loading into horizontal tension. Since the strength of the struts are higher under tensile loading, the black struts can enhance the overall mechanical performance of the unit cell in both tension and compression conditions.

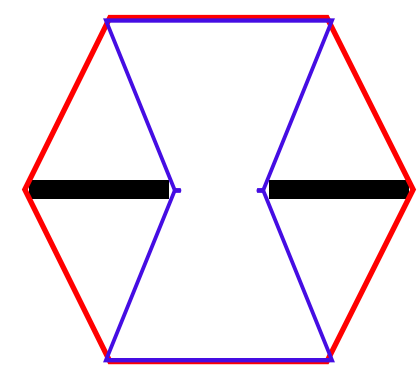

Figure 3: Schematically representation of the hybrid unit cell design.

The angle between the red inclined struts and vertical centerline $(\theta)$ of conventional honeycomb is defined as a design parameter for hybrid unit cell. Moreover, the corresponding angle of $\mathrm{AHC}$, the angle between the blue inclined struts and red inclined struts, is termed as another design parameter identified as $\gamma$. The detailed representation of the hybrid unit cell and its design parameters are shown in Figure 4.

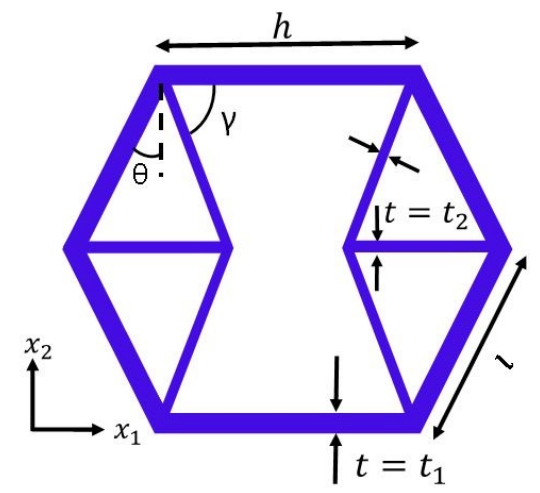

Figure 4: Design parameters of Hybrid honeycomb.

The parameter of $t_{1}$ is corresponding to the equal thickness of the struts with conventional honeycomb parent (two horizontal and four outer inclined struts). Also, the parameter $t_{2}$ is representing the thickness of the interior struts, for inclined struts from the auxetic parent and two horizontal struts connecting the elbows.

In addition, the analytical expression of the hybrid unit cell's relative density (for small values of $t_{1} / l$ and $t_{2} / l$ ) is represented in (4) based on its six design variables.

$$
\frac{\rho^{*}}{\rho_{s}}=\frac{3 t_{1} \sec (\theta)+2 t_{2}[\operatorname{Cot}(\gamma)+2 \csc (\gamma)+\tan (\theta)]}{2[h+l \sin (\theta)]}
$$

\section{Finite element method simulation}

Finite element model of the 3D printed sample are created using LSprepost software. The CAD model of the unit cells with design parameters reported in Table 1 along with the material properties of the ABS-P430TM provided in Table 2 beside the tangential modulus of $50 \mathrm{MPa}$ are utilized to carry out the numerical simulation of the quasi-static compression tests. The bottom end of the models are supported using a fixed rigid plate (corresponding to the lower fixture of the UTM) and the loading is applied via another rigid plate with prescribed displacement (corresponding to the upper fixture of the UTM) from the top end. The finite element model of a unit cell with corresponding boundary and loading conditions is shown in Figure 5.

LS-Dyna commercial explicit code is employed to run the numerical simulations. The numerical simulations were carried out using commercial FEA software LS-Dyna. The hexahedron element with hourglass control and nominal size of $2 \mathrm{~mm}$ is used to mesh to unit cell models. In addition, the penalty surface to surface contact algorithm is applied to prevent interpenetration during the deformation of the structures. 


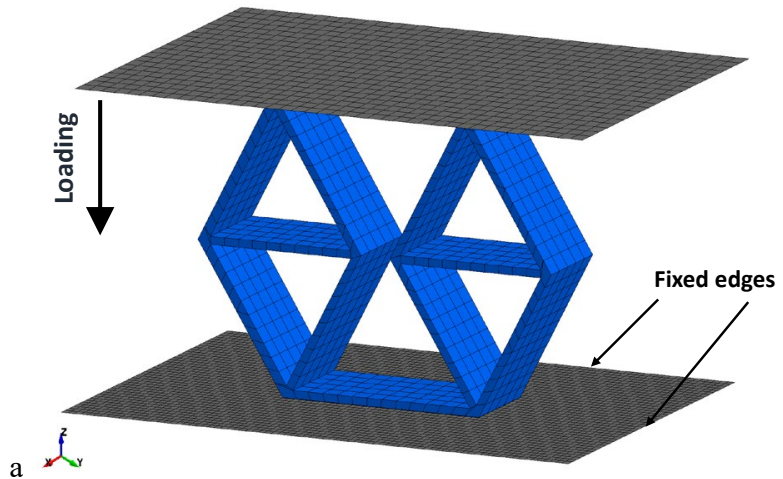

Figure 5: Finite element model of the HHC unit cell with loading direction and boundary condition.

\section{Sample preparation and testing}

Three different unit cells are created using the design parameters discussed in the methodology section. Unit cells of each model are precisely designed based on the (1) and (4) , using SolidWorks software (Dassault Systèmes). The Computer-Aided-Design (CAD) model of the unit cells, manufactured sample, and their corresponding design parameters are reported in Table 1.

Table 1: Different unit cells and their design parameters.

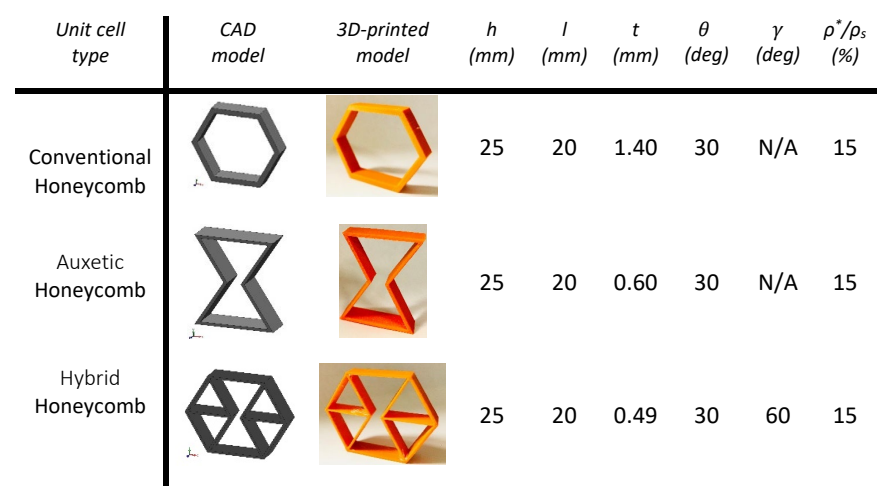

These samples are produced using Fused Deposition Modeling (FDM) process. The build material Acrylonitrile Butadiene Styrene (ABS) polymer is used to manufacture the samples using Stratasys U-print 3D-printer machine. The material properties of ABS-P430 are reported in Table 2. The high-resolution stereo lithography (STL) files are created from the CAD models and fed into 3D-printer. The layers resolution for additive manufacturing is set to the 0.01 inch along with solid infill feature to enhance the printing quality. The manufactured unit cells, as well as the CAD models, are shown in Table 1. Three samples of each unit cell are created along the z-direction as the build direction to improve the finishing and avoid using unnecessary support material in the different unit cells with complex structure. The designed unit cells are further utilized as the core material to design sandwich structures. The different unit cells are linearly patterned and assembled beside one another to create the sandwich structures with a uniform cellular core.

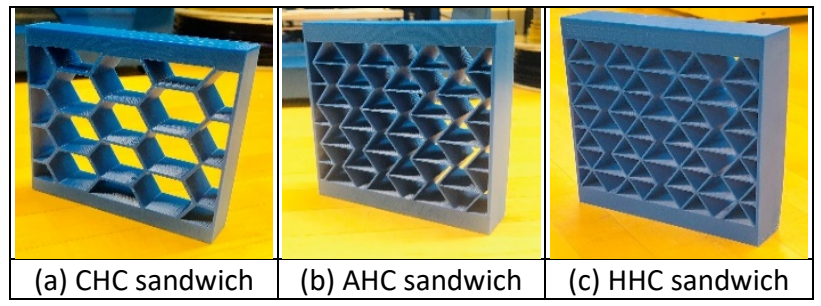

Figure 6: Sandwich structures with different core designs.

The sandwich structures with different core designs are 3D-printed using the same material as used to fabricate the unit cells (ABS-P430). Three sandwich designs are generated based on the described unit cells in the methodology section shown in Figure 6. They are conventional honeycomb sandwich, auxetic honeycomb sandwich, and Hybrid honeycomb sandwich structures. The upper layer, the low layer and the side wall of three sandwich structures have the same thickness values to maintain the consistent volume fraction. The thickness of the upper and lower layers is $15 \mathrm{~mm}$ and the side walls thickness is 2.25 $\mathrm{mm}$.

Table 2 : Mechanical properties of ABS-P430

\begin{tabular}{|c|c|c|c|c|c|}
\hline Material & $\begin{array}{c}\text { Elastic } \\
\text { modulus } \\
\left(E_{s}\right)\end{array}$ & $\begin{array}{c}\text { Yield } \\
\text { strength } \\
\left(\sigma_{y}\right)\end{array}$ & $\begin{array}{c}\text { Poisson's } \\
\text { ratio } \\
(v)\end{array}$ & $\begin{array}{c}\text { Ultimate } \\
\text { strength } \\
\left(\sigma_{U l}\right)\end{array}$ & $\begin{array}{c}\text { Density } \\
\left(\rho_{s}\right)\end{array}$ \\
\hline $\begin{array}{c}\text { ABS- } \\
\text { P430 }\end{array}$ & $\begin{array}{c}2.2 \\
(\mathrm{GPa})\end{array}$ & $\begin{array}{c}31 \\
(\mathrm{MPa})\end{array}$ & 0.35 & $\begin{array}{c}33 \\
(\mathrm{MPa})\end{array}$ & $\begin{array}{c}1.04 \\
(\mathrm{~g} / \mathrm{cm} 3)\end{array}$ \\
\hline
\end{tabular}

The thickness value of the upper and lower shell of the sandwich is $1.5 \mathrm{~mm}$. The 3D-printed sandwich structures are shown in Figure 6. The in-plane compressive tests are conducted using ETM Universal Testing Machine (UTM- 5KN) with loading rate of $1 \mathrm{~mm} / \mathrm{min}$. Forcedisplacement data captured by the test machine are the calculated nominal stress and nominal strain for each experiment. Three experimental tests are reported for each unit cell as well as FEA results.

\section{Results and discussion}

This section of the paper reports the structural performance of the different unit cells: the conventional honeycomb ( $\mathrm{CHC})$, the auxetic honeycomb (AHC), and the hybrid honeycomb (HHC). The mechanical performance of these designs is studied through the corresponding strain-stress behavior obtained from experimental tests as well as numerical simulations. For experimental results, three samples of each unit cell are 3D-printed and tested under the experimental setup described in the previous section. Moreover, the numerical results are calculated through the reaction force and 
resultant displacement of the rigid wall provided by the explicit finite element code.

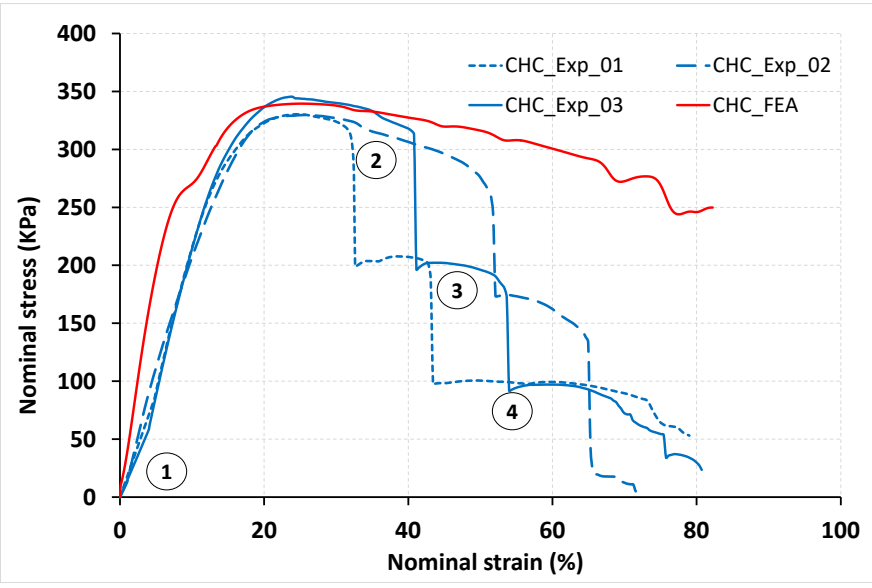

Figure 7: Stress-strain curve for experimental tests and numerical simulation of $\mathrm{CHC}$ unit cell.

The experimental results of the conventional honeycomb samples subjected to the uniaxial compression demonstrate in Figure 7. The different samples produce similar stress-strain plots with multiple sequential failures for each individual unit cell. The unit cells remain in the elastic deformation mode for the strain below $10 \%$ followed by a plateau stress of $340 \mathrm{KPa}$ (numerical average of three experiments) in range of $15 \%$ to $35 \%$ of strain. At this range of strain, the samples undergo elasto-plastic deformations due to bending of inclined struts.

The further increase in the nominal strain leads to the first failure of the conventional honeycomb samples. This failure is due to the initiation of the plastic regions at the corners with inclined edges shown in state 2 of the specimen in Table 3 . Congruently, the nominal stress values in Figure 7 suddenly drop to $300 \mathrm{KPa}$ due to the formation of these plastic hinges.

After the failure of the outer corners, the specimen maintains the energy absorbing for a short range of strain (5\%-10\%) through further bending the inclined struts and deformation in horizontal ones. The deformations occur within this short range lead to the second failure in the sample observed as a further drop in the stress value at stage 3 (Figure 7). The initialization of the failure at of the right corner between the bottom and the inclined strut is determined to be the prominent cause of the corresponding failure and drop in the stress value to $100 \mathrm{KPa}$. In final the stage of the stress-strain behavior (stage 4), the specimen absorbed the energy until the separation of the bottom strut and the connecting inclined strut. This separation leads to the collapse of the unit cell and maintains the stress value below $10 \mathrm{KPa}$.

Focusing on the numerical simulation of the corresponding compression test, the numerical stress-strain behavior of the specimen confirms the experimental stress-strain behavior in the elastic region (Figure 7). However, as the numerical solution enters into the elastoplastic region, the specimen goes under the plastic deformation without any significant structural failure. The absence of any structural failure reflected the accurate behavior of the preliminary numerical simulation without any failure criteria for the material model. Altogether, this behavior caused the insignificant drop in the stress value after state 2 until the specimen reaches the maximum deformation (Figure 7). This overall elastic as well as elasto-plastic behavior is shown through several stages in Table 3. In sum, the experimental test results and numerical results of the conventional honeycomb specimen showed a great deal of agreement in the corresponding stress-strain behavior. However, the inclusion of the failure criteria of the material model in the numerical solution is still under the investigation for the enhancement of the reliability of the numerical simulation to predict the accurate and actual behavior of the conventional honeycomb specimen.

Table 3: Sequence of deformed configurations during deformation and failure of $\mathrm{CHC}$ unit cell.

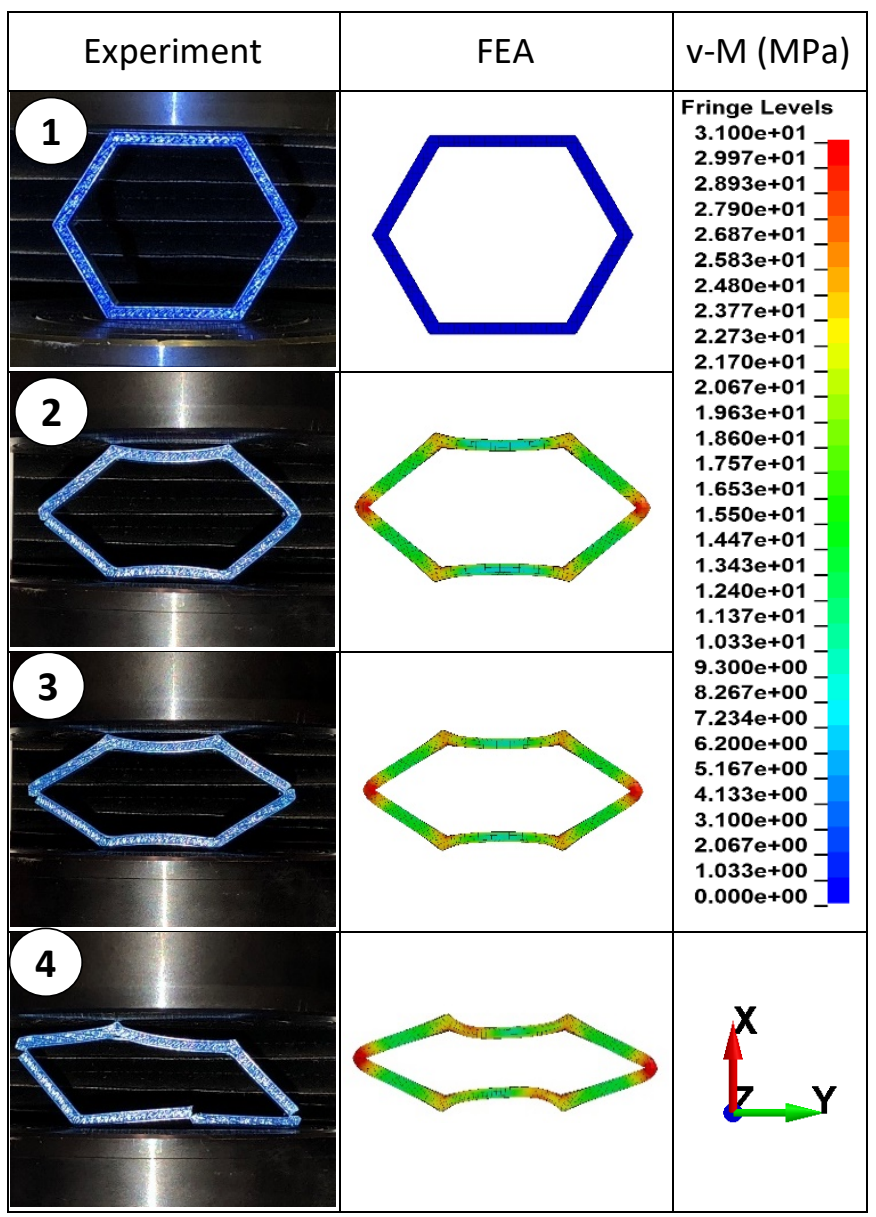

The results of the auxetic unit cell design for three experiments along with the numerical simulation are shown in Figure 7. The samples under the uniaxial compression loading indicate a similar behavior. The stage 1 in Figure 8, clearly represents the bending of inclined struts of AHC unit cell with no plastic failure and/or contacts between the struts, matching state 1 of Table 4 . The stage one is similar to the elastic region with no permanent failure. Increasing the strain up to $15 \%$ start the contact between the internal corners of the AHC unit cell. The contact force between the struts forms local plastic failure in that region (state 2 of Table 4) as well as a sharp increase in the stress value to beyond $100 \mathrm{KPa}$. The inclined struts undergo compressive buckling load and bending. As it is shown in stage 3 of Figure 8, the plastic hinge starts at one of the inclined struts.

Page 5 of 8 


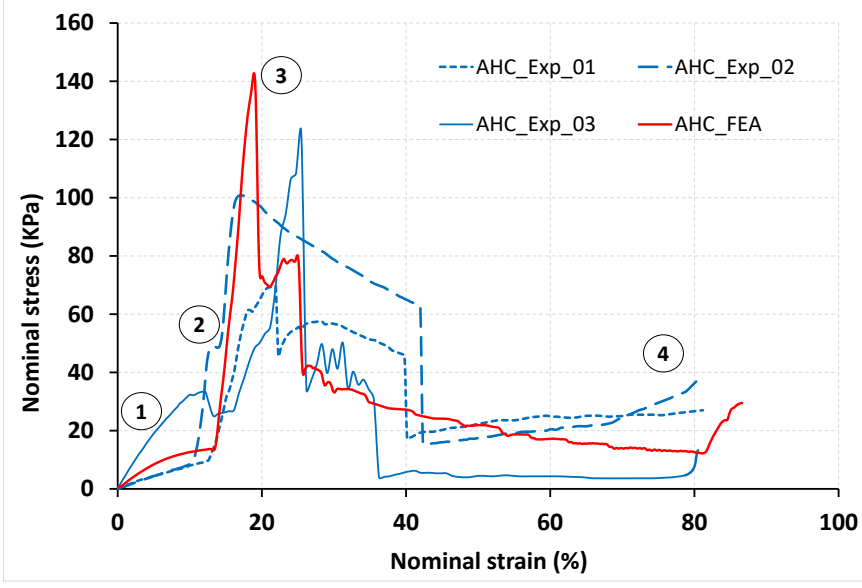

Figure 8: Stress-strain curve for experimental tests and numerical simulation of AHC unit cell.

Two types of failure are observed in experiments. First, the hinge appears far from the contact region, and the corners lose the contact. This brittle failure is followed by a steep drop in nominal stress observed as experiment 3 and FEA in Figure 8. This phenomenon is clearly observed in state 3 of the Table 4 . Second, the contact force between the corners gradually decrees and the hinge is very close to the contact region. This type of failure results in a slight decrease in stress value and persisting of the bending deformation in the inclined struts. The second type of failure is observed in experiments 2 and 3. The gradual drop of the stress is shown in Figure 8. At the strain values beyond $80 \%$, the unit cell collapses completely, and the hardening phase starts corresponding to stage 4 .

Hybrid honeycomb (HHC) unit cell indicates a unique stress-strain relation as compared to the auxetic and conventional honeycomb ones. The mechanical behavior of the HHC unit cells investigated using three experimental tests and the numerical simulation using FEA. Figure 9 shows the plots of the nominal stress versus nominal strain for physical tests and the simulation. The experimental tests and FEA indicate four different stages for mechanical behavior of HHC unit cell. The stage one shows a linear-elastic behavior similar to a solid material with the complete strain recovery. As the compressive strain increases, the nominal stress raises to the peak level as presented in the plot (stage two of the Figure 9). The plastic collapse of the unit cell initiates due to the symmetrical bending of internal inclined struts.

Two different types of collapse are possible for the HHC unit cell under compressive loading. First, pure bending of the inclined struts with local plastic hinges (struts of group-a in the FEA model of Table 5). Second, the bending of the inclined struts combined with delamination of the neighboring struts due to manufacturing defects (state 2 of Table 5: experiment section). Consequently, the peak value of the stress depends on the collapse type. If a unit cell fails under the first type (pure bending with plastic hinges) the compressive strength of the structure is expected to go beyond $420 \mathrm{KPa}$ (peak values of experiment 3 and FEA in Figure 9). On the other hand, the cells fail with second collapse condition (bending combined with delamination) the compressive strength of the unit cells remains slightly below 350 $\mathrm{KPa}$ (peak stress values of experiment 1 and experiment 2 in Figure 9). of AHC unit cell.

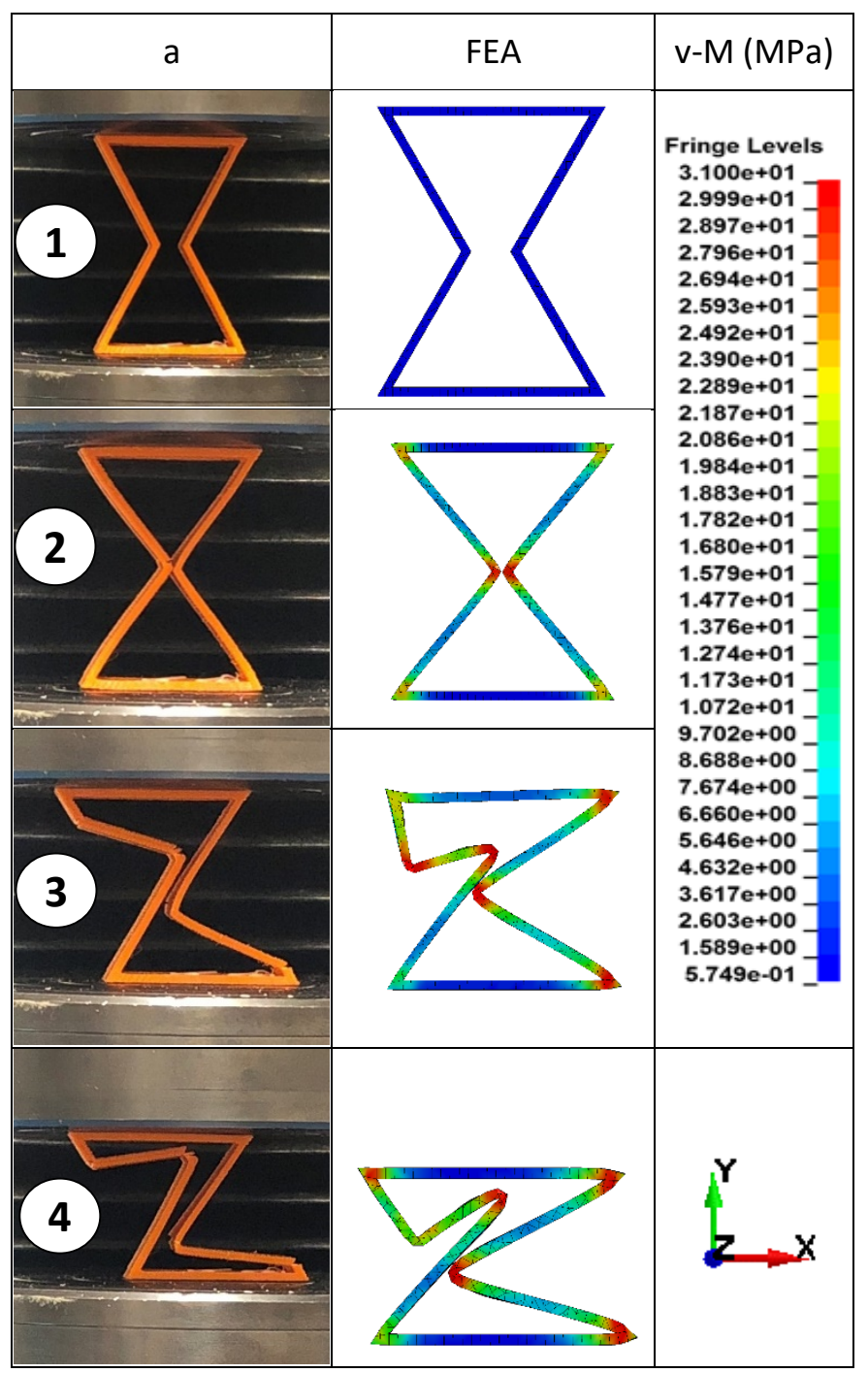

The initiation of the collapse in a unit cell leads to a substantial decrease in the nominal stress level. The collapse of the cell is further continued by growing the local plastic failure in the bent struts (struts of group-a in the FEA model) along with producing tensile stress in their adjacent inclined struts (struts of group-b in the FEA model) while the rest of the structure remains undamaged. As it is shown in state 3 of Table 5, the two undamaged triangles rotate up to 30 degrees (clockwise or counter-clockwise, depending on the location of the bent struts) due to the plastic deformation of the bent struts. The stress level of the structure gradually decreases from $150 \mathrm{KPa}$ to $50 \mathrm{KPa}$ while the unit cell is under rotational deformation mode (stage 3 in Figure 9). The rotational deformation places the internal inclined struts of the undamaged triangles in a vertical position and parallel to the loading direction (struts of group-c in the FEA model). The nominal stress increases moderately since the vertical struts perform resembling the columns under the compression loading. Stage 4 of Figure 9 illustrates the effect of the group-c struts buckling on the rise of the nominal stress. The second phase of the energy absorption with comparably high-stress values (about half of the peak stress value) initiates at the strains beyond $65 \%$. This high-stress value leads to the buckling of the 
group-c struts, succeeding the complete collapse of the unit cell and starting a hardening phase.

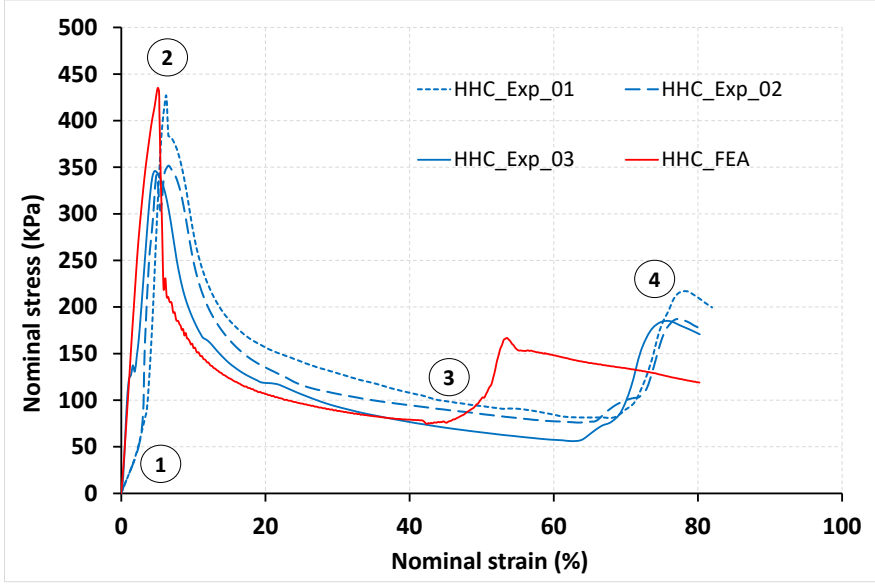

Figure 9: Stress-strain curve for experimental tests and numerical simulation of HHC unit cell.

Table 5: Sequence of deformed configurations during deformation and failure of HHC unit cell

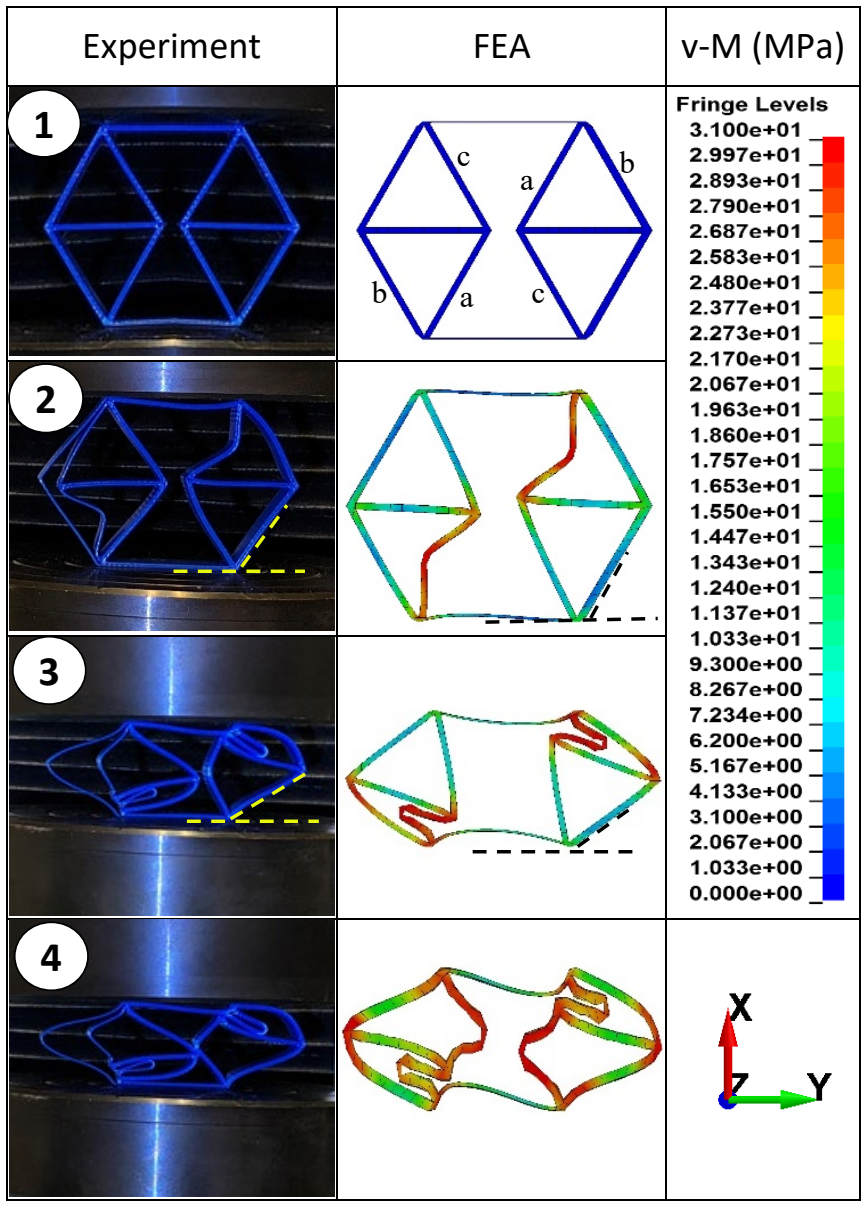

Page 7 of 8
Compressive strength is one of the most important characteristics of the cellular materials. Compressive strength is defined as the stress value at the yield point if the yield occurs before $10 \%$ strain. In the absence of such a yield point, the stress at $10 \%$ is considered as the compressive strength of the cellular material [19]. As another measure of structural performance, compressive strengths of the conventional honeycomb, auxetic honeycomb, and hybrid honeycomb samples are determined through the corresponding experimental stress-strain behavior (Figure 7 to Figure 9). The numerical values of the compressive strengths of the unit cell samples are shown in Table 6.

Table 6: Compressive strength of different unit cells.

\begin{tabular}{|l|c|c|c|}
\cline { 2 - 4 } \multicolumn{1}{c|}{} & \multicolumn{3}{c|}{ Compressive Strength (KPa) } \\
\cline { 2 - 4 } \multicolumn{1}{c|}{} & CHC & AHC & HHC \\
\hline Test 1 & 215.11 & 8.08 & 425.74 \\
\hline Test 2 & 210.45 & 8.94 & 351.59 \\
\hline Test 3 & 216.96 & 32.34 & 345.60 \\
\hline
\end{tabular}

The HHC unit cells has the highest compressive strength among all the three designs. Whereas, the AHC samples have the lowest compressive strength. The maximum compressive strength of the $\mathrm{CHC}$ design is $216.96 \mathrm{KPa}$ and is measured from the third experiment. However, this compressive strength of the $\mathrm{CHC}$ is $58 \%$ less than the minimum measured compressive strength of HHC unit cell.

The error bars shown in Figure 10, represents the uncertainty of the compressive strength of the samples based on the standard deviation. The compressive strength values of the $\mathrm{CHC}$ sample has the highest certainty, compared to the HHC design with the minimum uncertainty. This is due to the manufacturing defects in the structure. The thickness of the HHC unit cell is significantly less than CHC design $(0.49 \mathrm{~mm}$ compared to $1.4 \mathrm{~mm}$ ). Therefore, the structural integrity of the $\mathrm{HHC}$ sample is highly affected by the 3D-printer resolution.

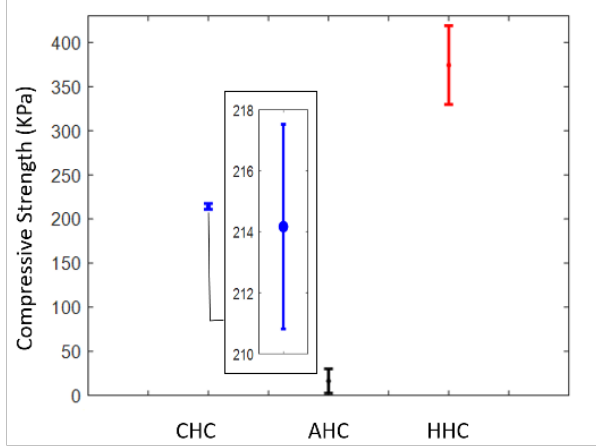

Figure 10: Uncertainty of the compressive strength in different designs.

\section{Conclusion}

In this study, a new cellular unit cell design was proposed for the core of the sandwich structure. The proposed unit cell, known as the hybrid honeycomb was designed by combining the conventional and auxetic honeycomb unit cell structures. The three designs of the cellular structures, conventional, auxetic and hybrid honeycomb were 3D- 
printed using FDM method with the same volume fraction of $15 \%$. The deformation modes and failure of the different unit cells were investigated using three quasi-statics compression tests and finite element analysis. The mechanical properties of the hybrid unit cell were compared to those of the conventional and auxetic unit cell structures. The compressive strength of the different design was measured using the experimental results. The new HHC unit cell design had a compressive strength $60 \%$ higher than the conventional honeycomb design.

\section{References}

1. Gibson, L.J. and M.F. Ashby, Cellular solids: structure and properties. 1999: Cambridge university press.

2. Allen, H.G., Analysis and design of structural sandwich panels: the commonwealth and international library: structures and solid body mechanics division. 2013: Elsevier.

3. Vinson, J., The behavior of sandwich structures of isotropic and composite materials. 2018: Routledge.

4. Schaedler, T.A. and W.B. Carter, Architected cellular materials. Annual Review of Materials Research, 2016. 46: p. $187-210$.

5. Hönig, A. and W.J. Stronge, In-plane dynamic crushing of honeycomb. Part I: crush band initiation and wave trapping. International Journal of Mechanical Sciences, 2002. 44(8): p. 1665-1696.

6. Damadam, M., R. Moheimani, and H. Dalir, Bree's diagram of a functionally graded thick-walled cylinder under thermo-mechanical loading considering nonlinear kinematic hardening. Case Studies in Thermal Engineering, 2018. 12: p. 644-654.

7. Zhang, X.-c., et al., Numerical Investigation on Dynamic Crushing Behavior of Auxetic Honeycombs with Various Cell-Wall Angles. Advances in Mechanical Engineering, 2015. 7(2): p. 679678.

8. Carneiro, V.H., J. Meireles, and H. Puga, Auxetic materials - A review. Materials Science-Poland, 2013. 31(4): p. 561-571.

9. MOHEIMANI, R., R. SARAYLOO, and H. DALIR. Symmetrical and Antisymmetrical Sequenced Fibers with Epoxy Resin on Rectangular Reinforced Structures Under Axial Loading. in Proceedings of the American Society for Composites-Thirty-third Technical Conference. 2018.

10. Ju, J. and J.D. Summers, Compliant hexagonal periodic lattice structures having both high shear strength and high shear strain. Materials \& Design, 2011. 32(2): p. 512-524.

11. Ingrole, A., A. Hao, and R. Liang, Design and modeling of auxetic and hybrid honeycomb structures for in-plane property enhancement. Materials \& Design, 2017. 117: p. 72-83.

12. Alderson, A. and K.L. Alderson, Auxetic materials. Proceedings of the Institution of Mechanical Engineers, Part G: Journal of Aerospace Engineering, 2007. 221(4): p. 565-575.

13. Buitrago, B.L., et al., Modelling of composite sandwich structures with honeycomb core subjected to high-velocity impact. Composite structures, 2010. 92(9): p. 2090-2096.

14. Lu, C., et al., Mechanical performance of $3 D$-printing plastic honeycomb sandwich structure. International
Journal of Precision Engineering and Manufacturing-Green Technology, 2018. 5(1): p. 47-54.

15. Sun, Y., et al., Bending behavior of composite sandwich structures with graded corrugated truss cores. Composite Structures, 2017.

16. Wu, Q., et al., A novel strengthening method for carbon fiber composite lattice truss structures. Composite Structures, 2016. 153: p. 585-592.

17. Zhou, H., et al., Mechanical performance and energy absorption properties of structures combining two Nomex honeycombs. Composite Structures, 2018. 185: p. 524-536.

18. Raeisi, S. and A. Tovar, The Effect of the Cell Shape on Compressive Mechanical Behavior of $3 D$ Printed Extruded Cross-sections. 2018, SAE Technical Paper.

19. International, A., Standard Test Method for Compressive Properties of Rigid Cellular Plastics. 2016, www.astm.org: West Conshohocken, PA.

\section{Contact Information}

\section{Sajjad Raeisi}

School of Mechanical Engineering

Purdue University, West Lafayette, IN sraeisi@purdue.edu

\section{Farha Deeba Ansari}

Department of Mechanical and Energy Engineering Indiana University-Purdue University Indianapolis. fdansari@iu.edu

\section{Prasad Balasaheb Tapkir}

Department of Mechanical and Energy Engineering Indiana University-Purdue University Indianapolis. pbtapkir@iu.edu

Andres Tovar (corresponding author)

Associate Professor

Department of Mechanical and Energy Engineering

Indiana University-Purdue University Indianapolis 723 West Michigan Street, SL 260N

Indianapolis, IN 46202-5132, USA

tovara@iu.edu 